\title{
INFLUÊNCIA DO MÉTODO DE SAPONIFICAÇÃO NA MICROFLOTAÇÃO DE APATITA UTILIZANDO ÓLEO DA CASTANHA DA MACAÚBA
}

\author{
André Carlos Silva ' \\ Elenice Maria Schons Silva ${ }^{\prime}$ \\ Thiago Felipe Vieira Silva ' \\ Kelly Cristina Ferreira ${ }^{2}$
}

\section{Resumo}

Dentre os minerais fosfatados, o grupo das apatitas é a principal fonte do fosfato usado como insumo na fabricação de fertilizantes agrícolas. A macaúba (Acrocomia Aculeata) é um fruto típico do cerrado brasileiro e apresenta grandes concentrações de componentes oleaginosos na castanha (36,2\%). O perfil de ácidos graxos da macaúba mostra que as concentrações mais elevadas na castanha são de ácido láurico $(39,7 \%)$ e ácido oleico $(25,8 \%)$. O presente trabalho procurou demonstrar, através de ensaios de microflotação em tubo de Hallimond, o potencial de utilização do óleo da castanha da macaúba na flotação de apatita. Os testes foram realizados com I g de apatita de alta pureza, numa granulometria de - I00 + I50\# (-I50+ I06 $\mu \mathrm{m})$, em valores de pH 8, 9 e 10 variando as concentrações do coletor em 2,5; 5,0; 7,5 e 10,0 mg/L. Os coletores usados foram o óleo da castanha da macaúba saponificado a quente $\left(90^{\circ} \mathrm{C}\right)$, a frio e o Flotigam 5806 da Clariant. Observou-se um aumento da recuperação de apatita utilizando o óleo saponificado a quente em relação ao saponificado a frio e, em alguns testes, também superior àquela obtida com o Flotigam 5806.

Palavras-chave: Flotação; Ácidos graxos; Óleo da castanha da macaúba.

\section{INFLUENCE OF THE SAPONIFICATION METHOD ON APATITE MICROFLOTATION USING MACAÚBA CHESTNUT OIL}

\begin{abstract}
Among the phosphorus bearing minerals, the apatite group is the main source of the phosphate used as input in the industrial fertilizers manufacture. Macaúba (Acrocomia Aculeata) is a tree, which naturally occurs in Brazilian's savanna and contains high concentrations of fatty acids in its nuts (36.2\%). The most abundant fatty acids in the nuts are lauric acid $(39.7 \%)$ and oleic acid (25.8\%). The present study sought to demonstrate, through microflotation experiments conducted in Hallimond tube, the potential use of macaúba oil in the flotation of apatite. The tests were performed with I $g$ of high purity apatite, in the particle size range of $-100+150 \#(-150+106 \mu \mathrm{m})$ at $\mathrm{pH}$ levels of 8,9 , and $10 \mathrm{~g}$ and concentrations of the collector of 2.5; $5.0 ; 7.5$ and $10.0 \mathrm{mg} / \mathrm{L}$. The collectors used were the macaúba's nut oil hot saponified at $90^{\circ} \mathrm{C}$ and at room temperature and Clariant's Flotigam 5806. The oil saponification at $90^{\circ} \mathrm{C}$ yielded higher recoveries than that at room temperature and in some tests, the performance was superior to that achieved Flotigam 5806.
\end{abstract}

Keywords: Flotation; Fatty acids; Macaúba's nut oil.

\section{INTRODUÇÃO}

Diversos são os produtos químicos utilizados no processo de flotação. Dentre eles pode-se citar os ácidos graxos, que são particularmente relevantes por sua responsabilidade na concentração de importantes minérios brasileiros, tais como os fosfatados do grupo apatita. Esses minérios representam a principal fonte de fosfato para produção de fertilizantes, um insumo agrícola de grande consumo em um país essencialmente agropecuário como o Brasil.

Segundo Souza e Fonseca [I], cerca de $80 \%$ das jazidas fosfáticas naturais (fosfatos) brasileiras, são de origem ígnea

'Universidade Federal de Goiás - UFG, Catalão, GO, Brasil. E-mail: ancarsil@ufg.br

${ }^{2}$ Universidade Federal de Minas Gerais - UFMG, Belo Horizonte, MG, Brasil. 
com presença acentuada de rocha carbonatítica e minerais micáceos, com baixo teor (é o conteúdo de fósforo de uma rocha ou concentrado), em geral expresso em $\left(\mathrm{P}_{2} \mathrm{O}_{5}\right)$, enquanto que em termos mundiais esse percentual está em torno de $17 \%$. O grupo da apatita $\left(\mathrm{Ca}_{5}(\mathrm{Cl}, \mathrm{F}, \mathrm{OH})\left(\mathrm{PO}_{4}\right)_{3}\right)$ representa a maioria dos minérios de fósforo das rochas fosfatadas e, os depósitos formados por esses minérios sílico-carbonatados possuem uma complexa mineralogia, contendo impurezas que influenciam sua recuperação nas usinas de beneficiamento.

Para Albuquerque [2] os metais alcalinos terrosos semelhantes presentes na estrutura cristalina destes minerais contribuem para a adsorção não seletiva dos agentes coletores. Neste contexto, a escolha do sistema de reagentes de flotação é fator determinante na eficiência deste processo.

De acordo com Rubio et al. [3] e Capponi et al. [4], no processo de flotação extender, óleos não polares são utilizados como tal ou na forma de emulsão (para aumentar sua difusão em solução aquosa) em concentrações da ordem

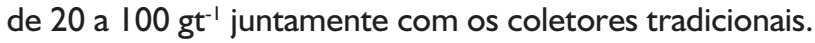
A palavra extender refere-se ao fenômeno de espalhamento do óleo em superfícies hidrofóbicas causando um aumento significativo na hidrofobicidade das partículas. Em relação aos microfenômenos que operam, é importante destacar que a característica mais marcante no uso de óleos, não é a adsorção e sim a "interação" entre gotas e partículas. Como as gotas são hidrofóbicas (portanto insolúveis em água), a interação ocorre por forças hidrofóbicas com as partículas que já possuem coletor e inclui etapas de colisão e adesão. Imediatamente após ocorrer a adesão, o óleo se "espalha" sobre a superfície da partícula, aumentando consideravelmente a hidrofobicidade. As mesmas podem, sob turbulência, se agregar e resolver o problema de tamanho e flotação incipiente.

De acordo com Tanaka et al. [5], a apatita apresentou valores de potencial zeta negativos e segundo os pesquisadores, os íons oleato adsorveram-se quimicamente, reagindo com o cálcio presente na superfície da apatita. Essa interação química foi mais forte e superou a repulsão eletrostática entre a superfície da apatita, carregada negativamente, e os íons oleato.

A finalidade básica do coletor é formar, seletivamente, uma camada hidrofóbica sobre uma superfície mineral, na polpa de flotação e, dessa forma, possibilitar condições para a fixação das partículas hidrofóbicas às bolhas de ar e a recuperação destas partículas no produto da espuma (Bulatovic [6]). Em geral, os coletores podem ser aniônicos (sulfidrílicos ou oxidrílicos) ou catiônicos (aminas e seus sais). Dentre os oxidrílicos, pode-se destacar a família dos carboxílicos, que contém os ácidos graxos, que apresentam grande destaque na literatura como coletores no processo de flotação. Estes podem ser encontrados em óleos de origem vegetal e animal, por exemplo (Baltar [7]).

De acordo com Guimarães et al. [8] certos vegetais, e gordura animal, são capazes de reagir glicerol e ácidos graxos neles contidos produzindo triaglicerol ou moléculas de óleo. Óleo extraído de vegetais é então purificado e submetido a um processo que combina aquecimento, alta pressão e alcalinidade, sendo convertido em ácido graxo. Os ácidos graxos são saponificados com $\mathrm{NaOH}$ para produzir sabões solúveis que agem como coletores de apatita. Alguns exemplos são castanha de babaçu, mamona, milho, oliva, arroz, farelo, soja, tall oil e sebo.

Dentre estes estudos cabe ressaltar o trabalho realizado por Costa [9], que analisou o uso de óleos vegetais amazônicos na flotação de minérios fosfáticos. Os resultados obtidos no trabalho indicam que é grande a possibilidade de utilização de óleos vegetais amazônicos como coletores na flotação dessa classe de minérios.

O uso dos óleos de mamona, coco, pequi e gergelim, saponificados, foi analisado por Vieira et al. [10] verificando-se que os dois últimos apresentaram bom desempenho como coletor, produzindo resultados semelhantes à flotação com oleato de sódio puro. No que tange a seletividade na flotação de minérios silico-carbonatados, pesquisas com o óleo de jojoba foram realizadas por Santos e Oliveira [I I] constatando a eficiência do óleo como coletor alternativo na separação de apatita e calcita.

De acordo com REMAPE [12] a macaúba é uma palmácea de ocorrência natural em toda a zona tropical da América Latina. Sua exploração econômica ocorre tanto em sistemas extrativistas quanto em cultivos racionais, com produtos e usos diversos como farmacológico, nutracêutico, madeireiro, artesanal, forrageiro, alimentício e combustível. Estima-se que sua produtividade de óleo aproxima - se à da palma africana (Elaeis guineensis), cerca de $5 \mathrm{t}$ de óleo por hectare. Esta é a cultura com maior produtividade de óleo por hectare cultivado e também a que contribui com a maior parcela do óleo vegetal produzido em nível mundial, sendo o custo médio por litro do óleo de US\$3,00.

\section{MATERIAIS E MÉTODOS}

As amostras de apatita utilizadas foram adquiridas de uma empresa de mineração e submetida, em laboratório, aos procedimentos de cominuição em moinho de bolas, peneiramento a úmido em um peneirador suspenso, filtragem a vácuo e posterior secagem em estufa com temperatura média de $60^{\circ} \mathrm{C}$.

Amostras deste mineral foram caracterizadas por espectrometria de fluorescência de raios-X na Anglo American Fosfatos utilizando-se um espectrômetro AXIOX MAX série DY $n^{\circ} 500$ I da Panalytical, e por difração de raios- $X$ em um Miniflex II da Rigaku, operando a 30 kV e 15 mA, usando tubo de cobre, radiação $\mathrm{Cu} \mathrm{K}(\alpha)$ e monocromador de grafite pirolítico. O tempo de contagem para as medidas foram de 2 segundos com passo angular de $0.02^{\circ}(2 \theta)$. Imagens digitais foram obtidas utilizando-se um estéreo microscópio LABTZ2250 da Laborana e um MEV da Jeol modelo JSM-66 I0, equipado com EDS da Thermo scientific NSS Spectral Imaging. Para a determinação do potencial 
Zeta da apatita foi utilizado um Zetasizer Nano da Malvern e solução de $\mathrm{NaCl}$ a $10^{-3} \mathrm{~mol} / \mathrm{L}$ como eletrólito indiferente.

O óleo da castanha da macaúba foi adquirido diretamente com os produtores, a UBCM (Unidade Beneficiamento Coco Macaúba) através da Associação Riacho D'antas Macaúba, com sede em Montes Claros (MG). O perfil de ácidos graxos do óleo da castanha da macaúba foi descrito por Silva et al. [13], no qual comparou-se o perfil encontrado com os resultados de outros autores, demonstrando que o perfil lípidico do óleo da castanha da macaúba muda de acordo com a região em que a planta é cultivada.

Para serem usados como coletores, os óleos foram submetidos à hidrólise alcalina, também chamada de saponificação. Esse procedimento permitiu torná-los solúveis em água, facilitando sua atuação como coletor. O procedimento de saponificação do óleo da castanha da macaúba foi feito a quente, no qual pesou-se $5 \mathrm{~g}$ do óleo a ser saponificado, adicionando-se $100 \mathrm{~mL}$ de solução alcoólica de $\mathrm{NaOH}$ (Álcool 95\% + $4 \mathrm{~g}$ de $\mathrm{NaOH}$ ), levou-se ao refluxo durante 2 horas em temperatura constante de $90{ }^{\circ} \mathrm{C}$, então evaporou-se o álcool durante 30 minutos, feito o sabão a solução intermediária usada na flotação foi feita pipetando-se $0,8 \mathrm{~mL}$ do saponificado em um balão de $200 \mathrm{~mL}$ e completando até o menisco com água de mesmo $\mathrm{pH}$ usado na flotação.

O procedimento de saponificação a frio do óleo castanha de macaúba (castanha) foi realizado seguindo as seguintes etapas: foram adicionados $5 \mathrm{~g}$ de óleo e $20 \mathrm{~g}$ de água, a mistura foi colocada em um agitador magnético, ligada a agitação, adicionou-se 7,5 mL de hidróxido de sódio a $10 \%$. Retornando o recipiente para a balança, adicionou-se água até que a solução atingisse $100 \mathrm{~g}$ e novamente o conjunto foi disposto no agitador magnético para homogeneização completa. No final do processo o produto tornou-se solúvel em água facilitando sua atuação como coletor. O Flotigam 5806 foi usado de maneira direta como coletor, não houve necessidade de nenhuma alteração.

O tubo de Hallimond foi o equipamento usado neste trabalho para determinar a flotabilidade (ou a recuperação) da apatita. Os testes de microflotação foram realizados com amostras puras de apatita, sendo as variáveis operacionais apresentadas na Tabela I. Como a água destilada possuía pH por volta de 7, o controle do $\mathrm{pH}$ foi realizado pela adição de ácido clorídrico e/ou hidróxido de sódio (ambos a 1\%) para

Tabela I. Condições dos testes de microflotação de apatita

\begin{tabular}{lc}
\hline \multicolumn{1}{c}{ Condições } & Valores \\
\hline Vazão de ar & $40 \mathrm{~cm}^{3} / \mathrm{min}$ \\
$\mathrm{pH}$ & 8,9 e I0 \\
Faixa granulométrica & $-100+150 \#(-150+106 \mu \mathrm{m})$ \\
Massa do mineral & $\mathrm{I} \mathrm{g}$ \\
Condicionamento & 7 minutos \\
Flotação & $\mathrm{I}$ minuto \\
Concentração de coletor & 2,$5 ; 5,0 ; 7,5$ e I0,0 mg/L \\
Coletores & Óleo saponificado a quente, \\
& saponificado a frio e Flotigam 5806 \\
\hline
\end{tabular}

se obter o $\mathrm{pH}$ desejado. Todos os testes foram realizados em triplicata, totalizando 36 testes.

O condicionamento da polpa foi realizado adicionando-se o mineral à $50 \mathrm{~mL}$ de solução coletora na parte inferior do tubo de Hallimond durante 7 minutos e sob agitação. Ao final do condicionamento completou-se o volume do tubo (320 mL) com água destilada no $\mathrm{pH}$ adequado ao ensaio. O tempo de flotação adotado foi de I minuto.

Testes de arraste hidráulico em Tubo de Hallimond visando mensurar o carreamento de partículas pelo fluxo ascendente gerado pela passagem das bolhas de ar foram realizados para a vazão de $40 \mathrm{~cm}^{3} / \mathrm{min}$.

\section{RESULTADOS E DISCUSSÃO}

A Figura la mostra resultados da microscopia ótica das amostras de apatita, onde pode-se perceber a presença de arestas vivas mesmo após a cominuição do mineral, percebe-se também sua cor azulada e transparência. As Figuras I b-d apresentam imagens de MEV com diferentes magnificações, nas quais observa-se que os cristais de apatita estão associados em pequenas quantidades a outros minerais. Tal fato fica evidente na Figura Id por se tratar de uma imagem de elétrons retro espalhados.

A Figura 2 mostra uma imagem da apatita, obtida através do MEV, assim como a sua análise por EDS, foram analisados 4 pontos de um dos grãos minerais. Através das análises de EDS é possível perceber que mesmo se tratando de uma apatita visualmente pura (com nenhuma ou pouca presença de outra espécie mineral), quando analisado através do EDS, percebe-se no ponto I (alaranjado) a presença de um mineral com presença de silício e sem flúor, portanto tratando-se de outro mineral que não seja apatita, possivelmente quartzo. Nos demais pontos os elementos se mantém os mesmos, caracterizando a fluorapatita, pela presença de flúor.

A análise de caracterização química por fluorescência de raios-X (Figura 3) da amostra de apatita revelou a presença de outros óxidos (potássio, silício e outros) em pequenas quantidades. Entretanto, as concentrações elevadas de $\mathrm{P}_{2} \mathrm{O}_{5}$ e $\mathrm{CaO}$ são condizentes com uma amostra de apatita com alto grau de pureza. A Figura 4 apresenta os resultados obtidos por difração de raios- $X$ das amostras de apatita, que indicaram a presença apenas de apatita, sendo que as demais contaminações não foram determinadas pelo ensaio.

A Figura 5 apresenta os resultados obtidos no ensaio de potencial zeta das amostras de apatita. Percebe-se que para os pHs 8 e 9 o potencial zeta da apatita medido foi de $-36,37$ e $-36,45$ respectivamente. Para o $\mathrm{pH} 10$ o valor esperado do potencial zeta é de aproximadamente $-38,50 \mathrm{mV}$. Para os $\mathrm{pH}$ s testados (variando de 4 a 12 ) não foi encontrado o ponto de carga zero (PCZ) da apatita.

Os testes de arraste hidráulico obtiveram recuperação média de aproximadamente $0,7 \%$ para a vazão de $40 \mathrm{~cm}^{3} / \mathrm{min}$. 


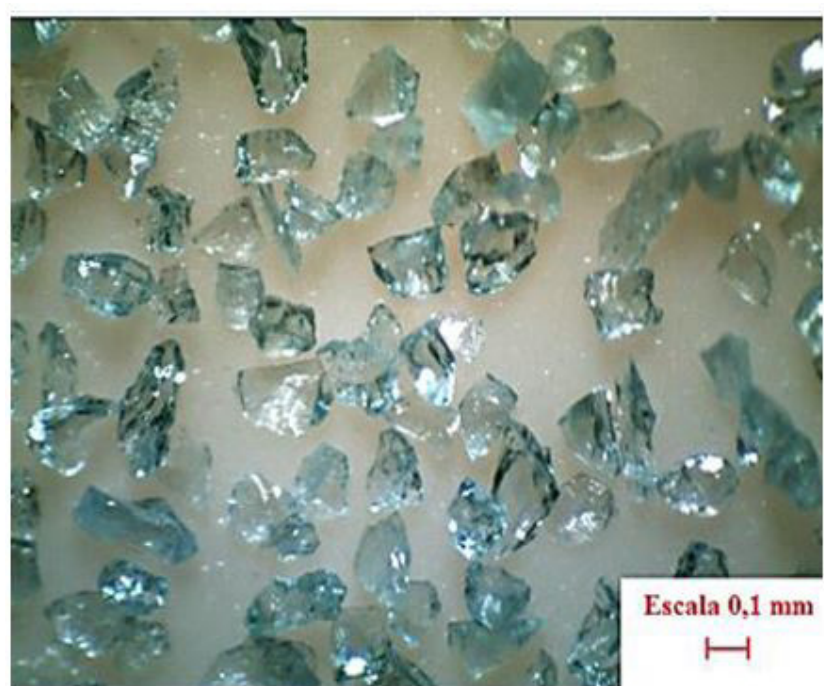

(a)

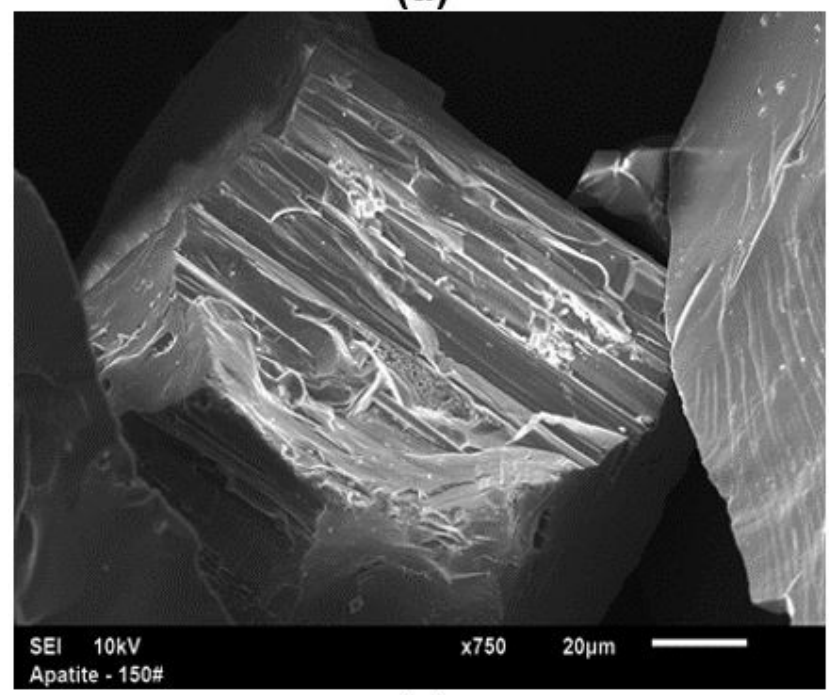

(c)

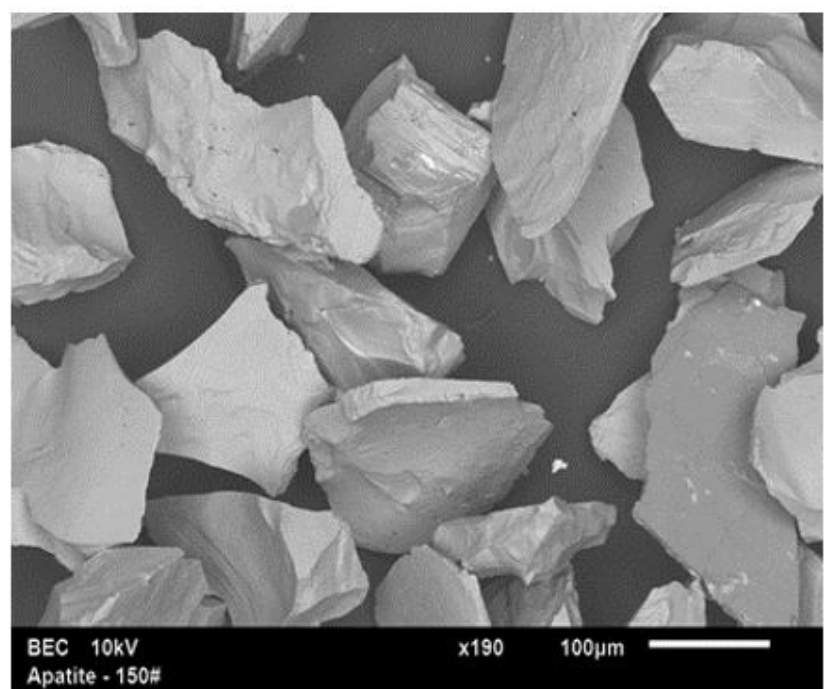

(b)

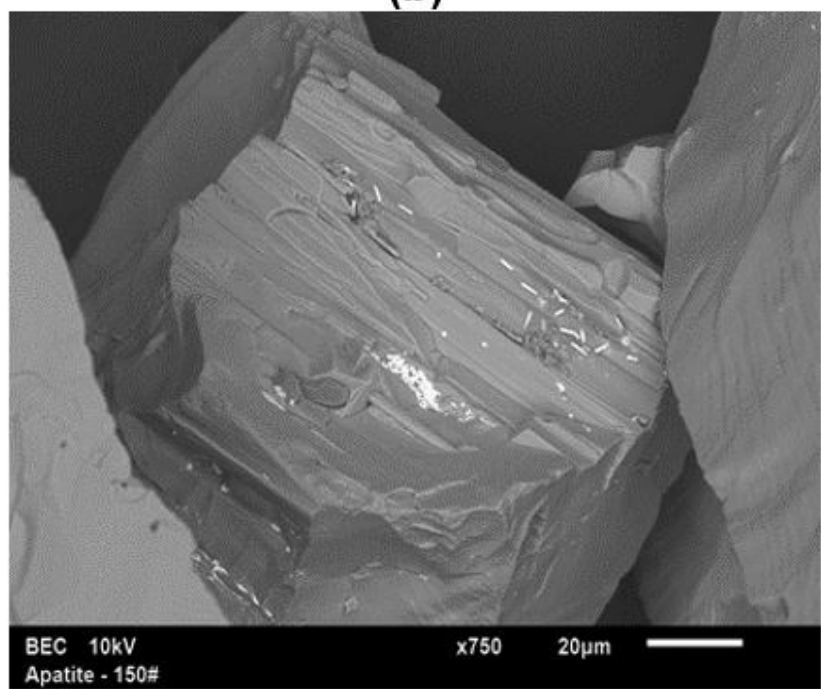

(d)

Figura I. (a) Resultado de microscopia ótica para amostras de apatita na granulometria de $-100+150 \#(-150+106 \mu \mathrm{m})$, de MEV SEI para apatita com magnificação de (b) xI 90 e (c) x750 e MEV BSE com magnificação x750 (d).

Desta forma os resultados dos testes de microflotação serão apresentados desconsiderando os valores de arraste.

Os resultados obtidos nos ensaios de microflotação foram condizentes com dados disponíveis na literatura para outros óleos (Costa [9], Silva et al. [13] e Silva et al. [14]). A Figura 6 mostra que para uma concentração de $2,5 \mathrm{mg} / \mathrm{L}$ - óleo da castanha da macaúba saponificado a quente possui uma recuperação $121,35 \%$ maior que o mesmo óleo saponificado a frio, porém ainda $25,83 \%$ menor que o Flotigam 5806. Para uma concentração de $5,0 \mathrm{mg} / \mathrm{L}$ ○ óleo saponificado a quente continua possuindo recuperação $58,19 \%$ maior que o óleo saponificado a frio e 1,86\% menor que o Flotigam 5806. Na concentração de 7,5 mg/L tanto o óleo saponificado a quente quanto o Flotigam 5806 possuem a mesma recuperação (95,5\%), $17,32 \%$ maior que a recuperação do óleo saponificado a frio. Percebe-se que todos os reagentes alcançam um patamar de recuperação, ou seja, ao chegar em determinada concentração o reagente mantém a sua recuperação quase que constante, mesmo que se aumente a concentração, para o Flotigam 5806 o patamar é alcançado na concentração de $2,5 \mathrm{mg} / \mathrm{L}$, no qual sua recuperação se mantém por volta de $92 \%$, o óleo saponificado a quente teve seu patamar em $5,0 \mathrm{mg} / \mathrm{L}$, porém apresentou uma redução na recuperação nas concentrações 7,5 e $10,0 \mathrm{mg} / \mathrm{L}$. O óleo saponificado a frio possui patamar em $7,5 \mathrm{mg} / \mathrm{L}$, com recuperação de $81 \%$, apresentando os menores resultados de recuperação nos testes.

A Figura 7 se assemelha à Figura 6, visto que o óleo saponificado a frio continua sendo o coletor que obteve as menores recuperações (mínimo de 30,62\% para 2,5 $\mathrm{mg} / \mathrm{L}$ e máximo de $83,94 \%$ para $10 \mathrm{mg} / \mathrm{L}$ ). Por outro lado, o óleo saponificado a quente, mesmo mantendo 
Silva et al.

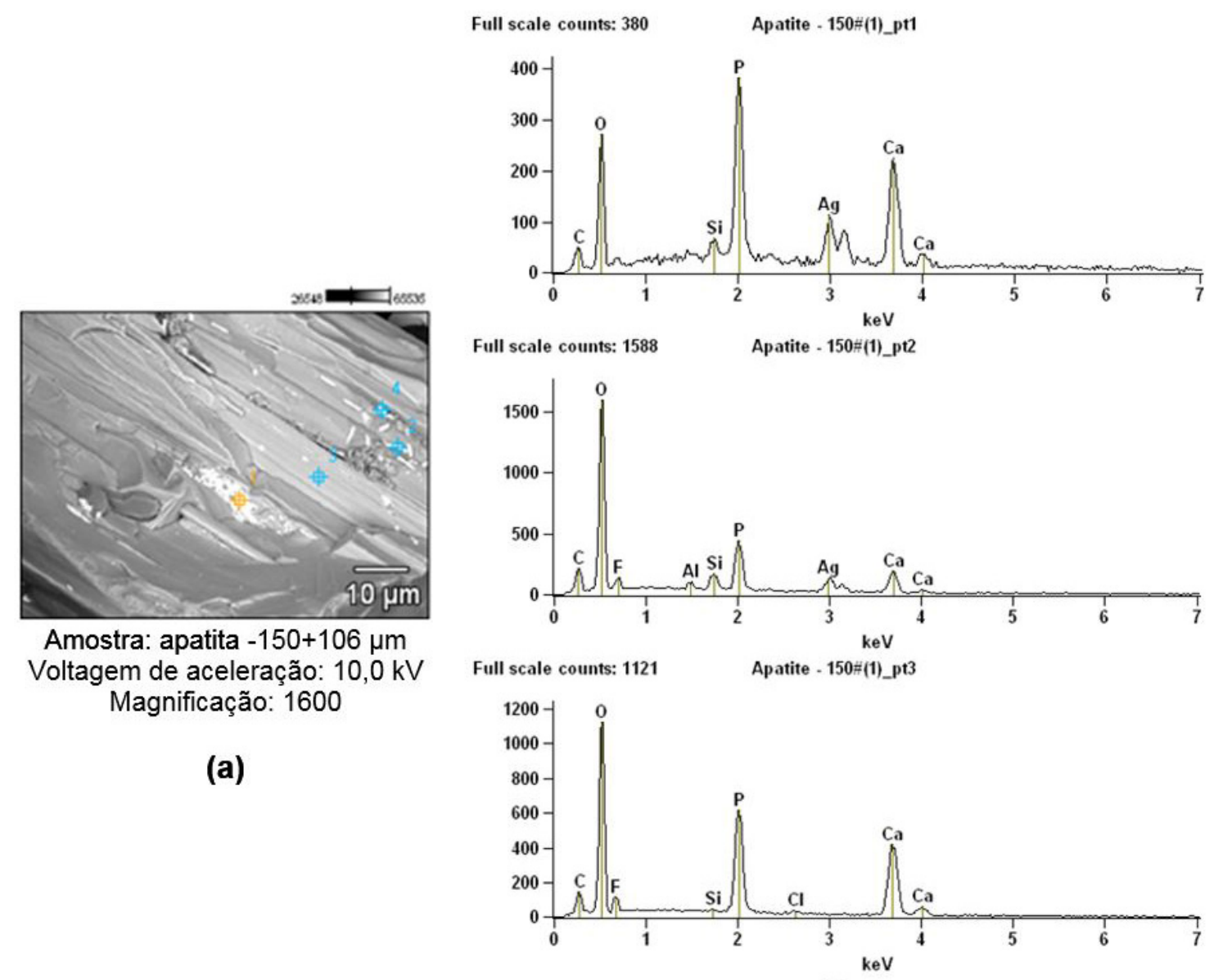

(b)

Figura 2. Resultados de EDS para uma amostra de apatita com granulometria - $150+106 \mu \mathrm{m}$ (a) imagem de MEV e (b) resultados de EDS para três diferentes pontos.

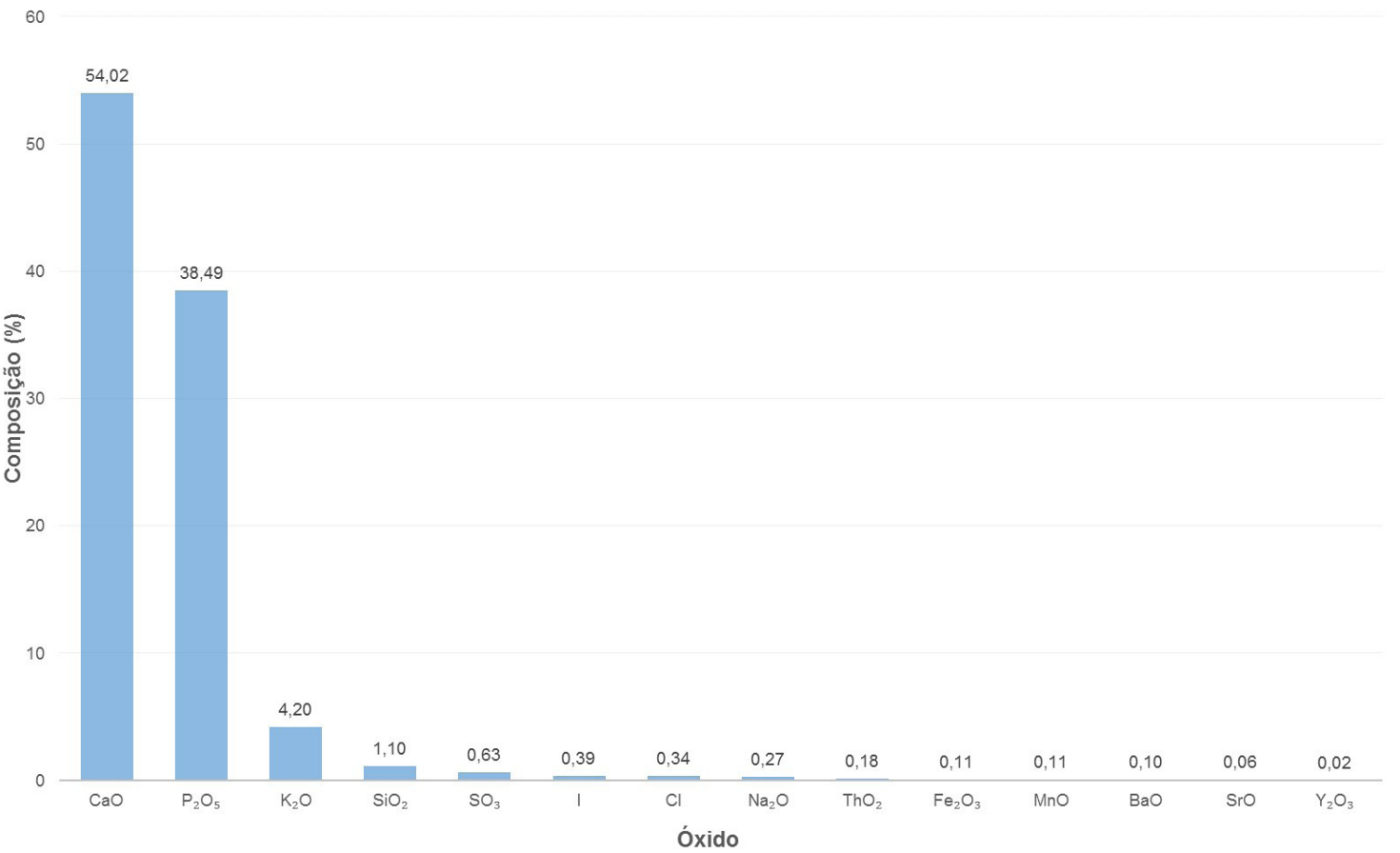

Figura 3. Resultados da análise da amostra de apatita por fluorescência de raios-X. 


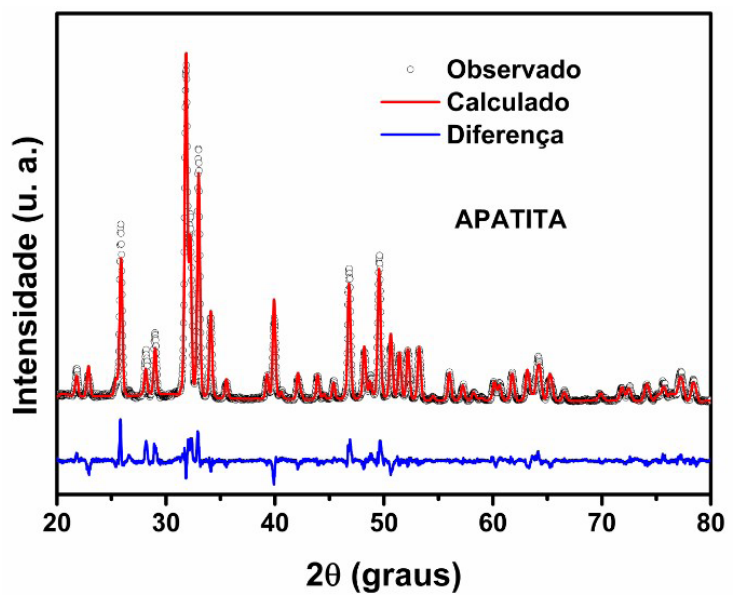

Figura 4. Resultados da análise da amostra de apatita por difração de raios- $X$. seu comportamento de redução de recuperação entre as concentrações 7,5 e 10 mg/L, apresentou na concentração de $7,5 \mathrm{mg} / \mathrm{L}$ recuperação $7,86 \%$ superior que a do Flotigam 5806. Nas demais concentrações testadas o óleo saponificado a quente se mantém com recuperação menor que a do Flotigam 5806. Os patamares para pH 9 têm uma variação maior que as observadas para $\mathrm{pH} 8$, porém as concentrações para alcançá-los se mantém.

$\mathrm{Na}$ Figura 8 observa-se uma recuperação muito inferior para o óleo saponificado a frio (recuperação máxima de $34,78 \%$ com concentração de $10 \mathrm{mg} / \mathrm{L}$ ). Já o óleo saponificado a quente se manteve com recuperação menor que o Flotigam 5806 para concentrações de 2,5 e 5,0 mg/L, porém superior ao mesmo para as demais concentrações testadas. O óleo saponificado a quente apresentou recuperação $4,90 \%$ maior em concentração $7,5 \mathrm{mg} / \mathrm{L}$ e $4,33 \%$ em

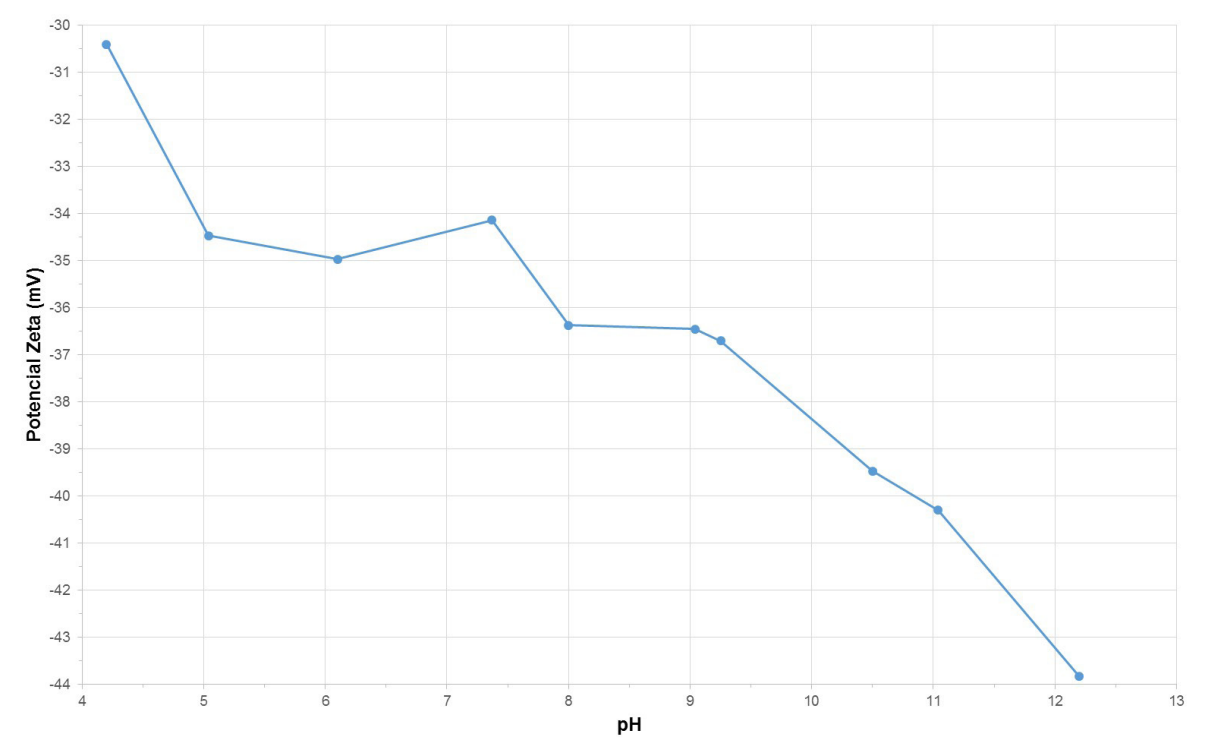

Figura 5. Resultados do potencial zeta das amostras de apatita.

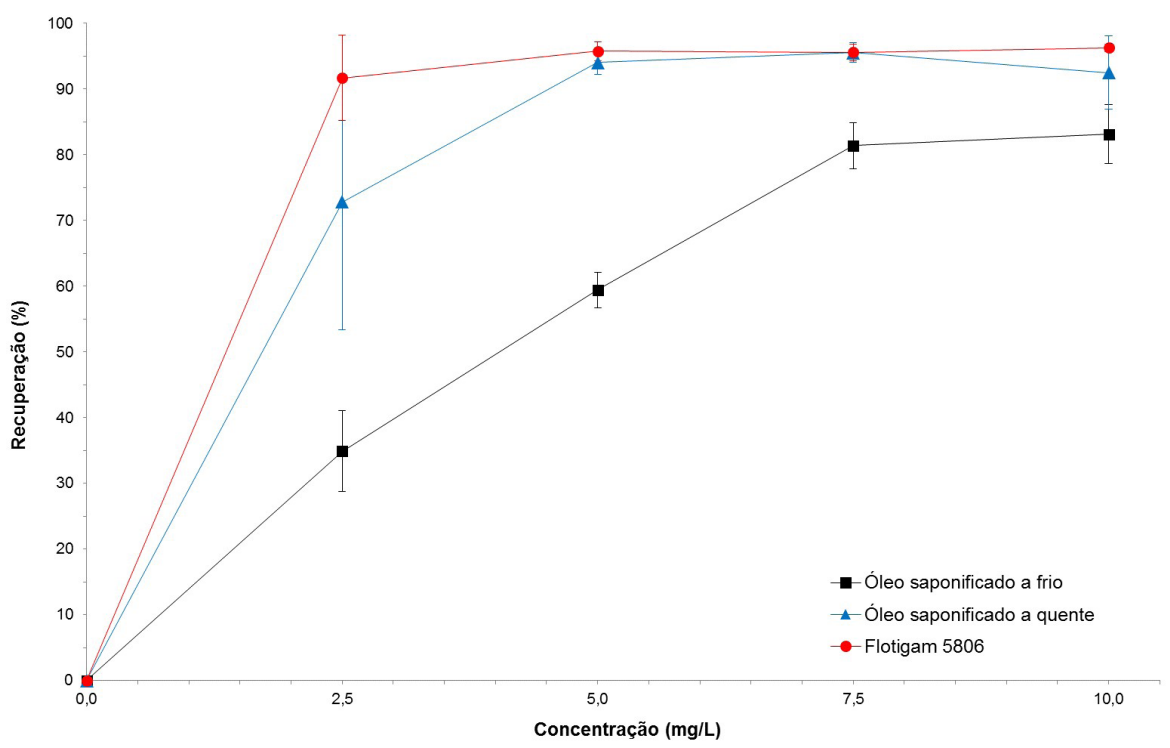

Figura 6. Recuperação de apatita em pH 8 para os coletores testados. 


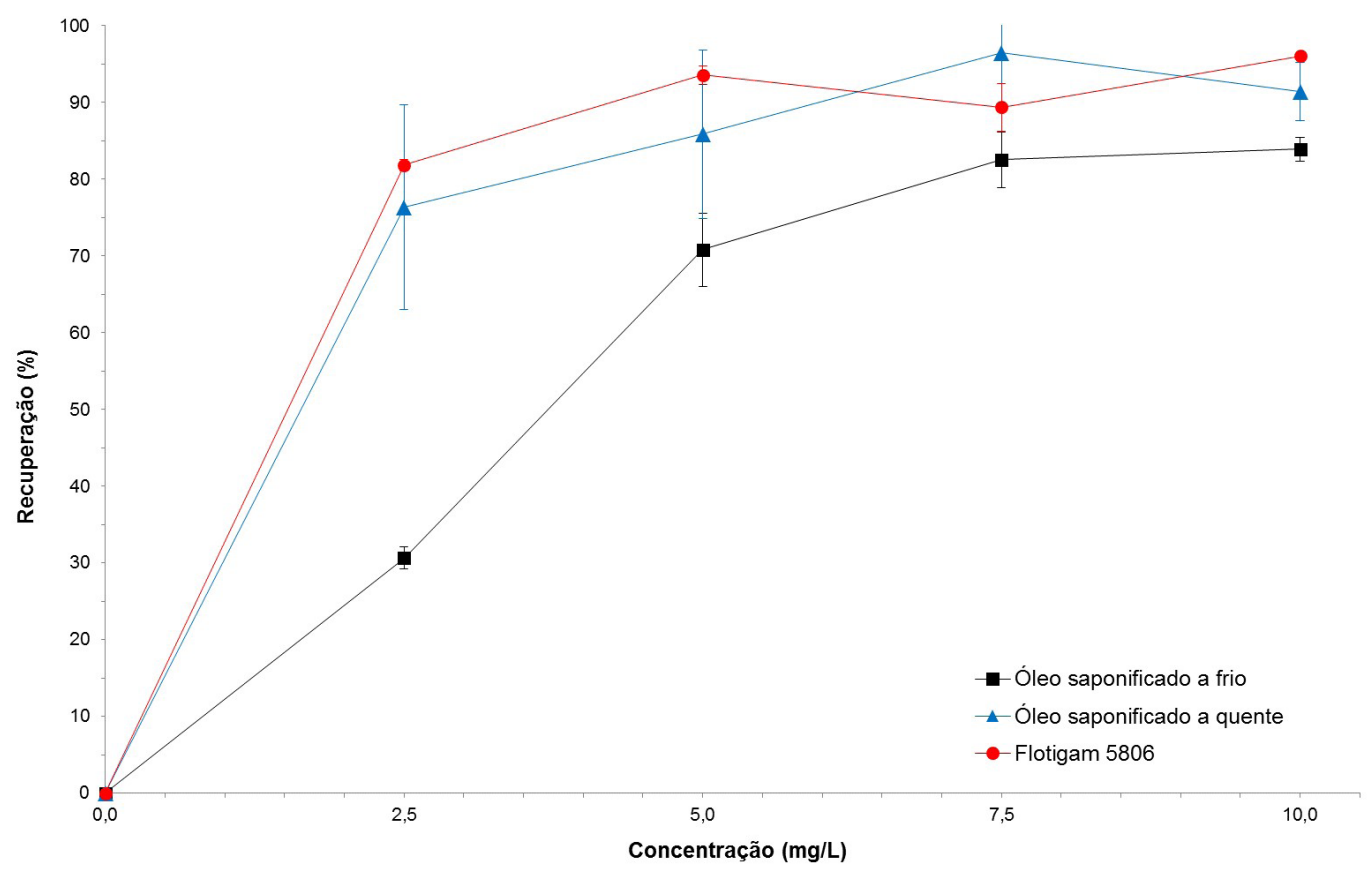

Figura 7. Recuperação de apatita em pH 9 para os coletores testados.

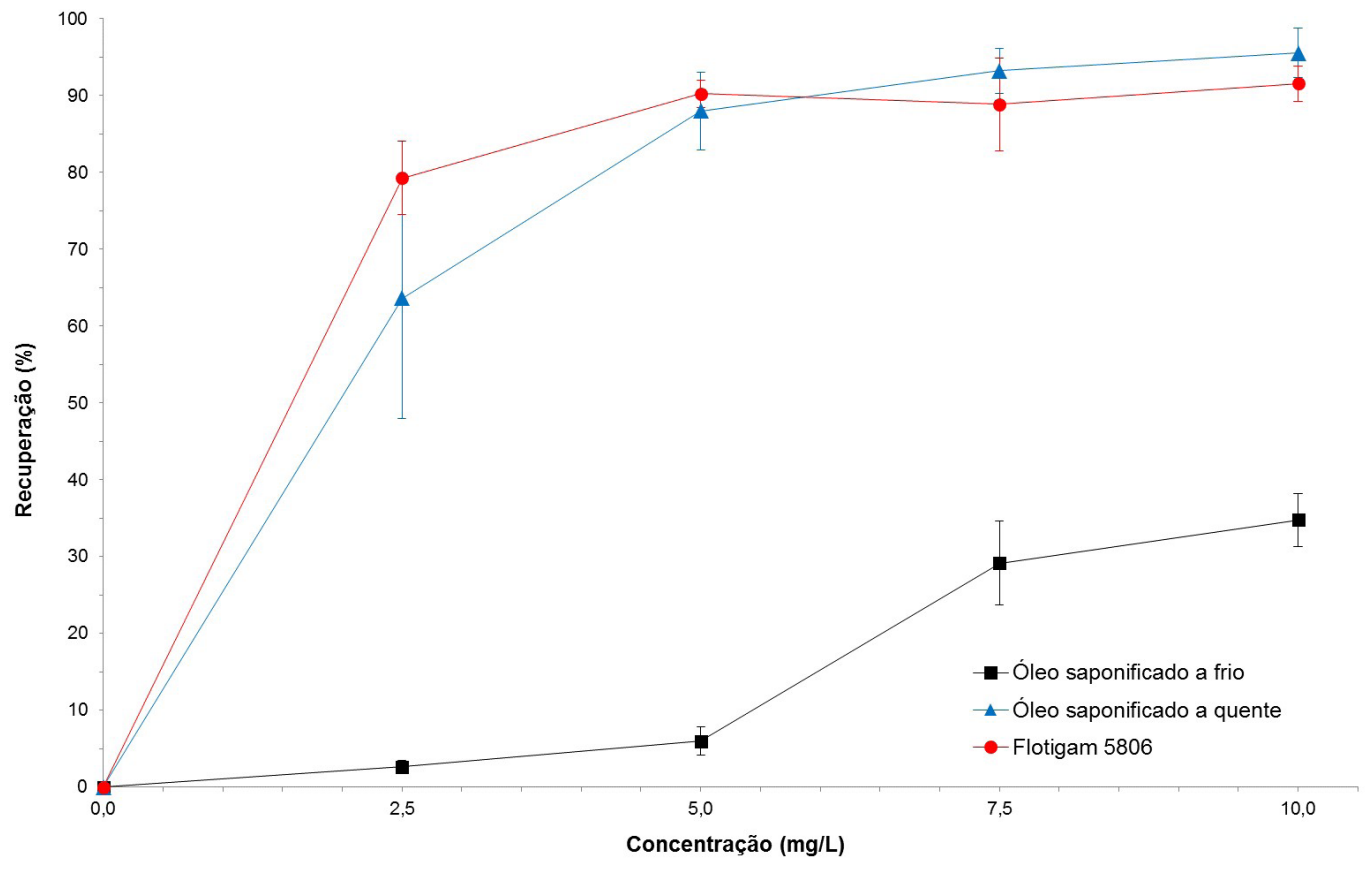

Figura 8. Recuperação de apatita em pH 10 para os coletores testados.

concentração $10 \mathrm{mg} / \mathrm{L}$. Os comportamentos tanto do Flotigam 5806 quanto para o óleo saponificado a quente são diferentes dos observados em $\mathrm{pH} 8$ e 9, visto que - Flotigam 5806 teve uma redução na recuperação entre as concentrações de 5,0 e $7,5 \mathrm{mg} / \mathrm{L}$ e se manteve inferior ao óleo saponificado a quente nas concentrações 7,5 e $10 \mathrm{mg} / \mathrm{L}$. O óleo saponificado a quente não apresentou a redução na recuperação apresentada nos $\mathrm{pHs} 8$ e 9 nas concentrações 7,5 e $10 \mathrm{mg} / \mathrm{L}$.

A Tabela 2 sumariza os melhores resultados encontrados para a recuperação de apatita em função dos pHs e concentrações de coletores testados. Nota-se que - óleo da castanha da macaúba, saponificado a quente, só obteve resultado superior ao Flotigam 5806 em três 
Tabela 2. Melhores resultados encontrados para cada pH e concentração de coletor testada

\begin{tabular}{|c|c|c|c|}
\hline pH & $\begin{array}{c}\text { Concentração } \\
(\mathrm{mg} / \mathrm{L})\end{array}$ & Coletor & $\begin{array}{c}\text { Recuperação } \\
(\%)\end{array}$ \\
\hline \multirow{4}{*}{8} & 2,5 & $\begin{array}{c}\text { Flotigam } \\
5806\end{array}$ & 91,7 \\
\hline & 5,0 & Flotigam 5806 & 95,8 \\
\hline & 7,5 & Flotigam 5806 & 95,6 \\
\hline & 10,0 & Flotigam 5806 & 96,3 \\
\hline \multirow{4}{*}{9} & 2,5 & Flotigam 5806 & 81,8 \\
\hline & 5,0 & Flotigam 5806 & 93,6 \\
\hline & 7,5 & Quente & 96,4 \\
\hline & 10,0 & Flotigam 5806 & 96,1 \\
\hline \multirow{4}{*}{10} & 2,5 & Flotigam 5806 & 79,3 \\
\hline & 5,0 & Flotigam 5806 & 90,3 \\
\hline & 7,5 & Quente & 93,2 \\
\hline & 10,0 & Quente & 95,5 \\
\hline
\end{tabular}

ensaios (em pH 9 e concentração de 7,5 mg/L e em pH 10 nas concentrações de 7,5 e $10,0 \mathrm{mg} / \mathrm{L}$ ).

\section{CONCLUSÕES}

Ensaios de microflotação em tubo de Hallimond com óleo da castanha da macaúba submetido a dois tipos diferentes de saponificação foram realizados e a recuperação de apatita foi medida para diferentes $\mathrm{pHs}$ e concentrações do óleo. Foi possível mostrar que a saponificação à quente aumenta significativamente a recuperação de apatita quando se utilizando o óleo da castanha da macaúba. Desta forma, o óleo da castanha da macaúba saponificado à quente se mostra como uma opção tecnicamente viável para substituir o Flotigam 5806 em condições de flotação de apatita com pH 10 e concentração do coletor variando entre 7,5 e $10,0 \mathrm{mg} / \mathrm{L}$. Fora dessas condiçóes o Flotigam 5806 obteve maiores recuperações.

Outro motivo para a adoção do óleo da castanha da macaúba deve-se ao fato do mesmo ser um reagente natural, sendo o Flotigam 5806 um coletor a base de óleo de soja com aditivos sintéticos.

Salienta-se a necessidade de realização de outros testes, para verificar sua aplicabilidade em escala industrial, pois a viabilização de um reagente mais seletivo na flotação e de baixo custo é particularmente interessante para o Brasil, pois poderia acarretar um melhor aproveitamento econômico e consequente aumento da vida útil das minas ou até mesmo viabilizaria novos empreendimentos, o que levaria a uma redução da importação de fertilizantes fosfatados.

\section{Agradecimentos}

Os autores agradecem às agências de fomento CNPq, CAPES, FAPEG e FUNAPE pelo suporte financeiro que possibilitou esta pesquisa. E adicionalmente, à Anglo American Fosfato e Universidade Federal de Goiás.

\section{REFERÊNCIAS}

I Souza AE, Fonseca DS. Fosfato. Brasília: DNPM; 2008. p. 546-568.

2 Albuquerque RO. Alternativas de processo para concentração do minério fósforo-uranífero de Itataia [tese de doutorado]. Belo Horizonte: Universidade Federal de Minas Gerais; 2010.

3 Rubio J, Capponi F, Matiolo E, Nunes D, Guerrero CP, Berkowitz G. Advances in flotation of mineral fines. In: Proceedings of the 22nd International Mineral Processing Congress; 2003 Sept 29-Oct I3; Cape Town. Johannesburg: SAIMM; 2003. p. 1002-1014.

4 Capponi F, Matiolo E, Rosa J, Rubio J, Berkowitz G, Pérez C. Avanços na flotação de finos de minérios sulfetados de cobre e molibdênio. In: Anais do XX Encontro Nacional de Tratamento de Minérios e Metalurgia Extrativa; 2004; Florianópolis, Brasil. Florianópolis, 2004. p. 69-77, vol. 2.

5 Tanaka Y, Katayama N, Arai S. Reagents in phosphate flotation. In: Somasundaran P, Moudgil BM. Reagents in mineral technology. New York: Dekker; 1988. chap. 20.

6 Bulatovic SM. Handbook of flotation reagents: chemistry, theory and practice: flotation of sulfide ores. Amsterdam: Elsevier Science \& Technology Books; 2007. vol. I.

7 Baltar C. Flotação no tratamento de minério. Recife: UFPE; 2008.

8 Guimarães RC, Araújo AC, Peres AEC. Reagents in igneous phosphate ores flotation. Minerals Engineering. 2004; 18(2):199-204.

9 Costa D. Uso de óleos vegetais amazônicos na flotação de minérios fosfáticos [tese de doutorado]. Belo Horizonte: Universidade Federal de Minas Gerais; 2012. 
Silva et al.

10 Vieira M, Lédo PGS, Lima RFS, Paulo JBA, Brandão PRG. Flotação de calcita a partir de óleos vegetais regionais saponificados como agentes coletores. In: Anais do $6^{\circ}$ Congresso Brasileiro de Engenharia Química em Iniciação Científica; 2005; Campinas, Brasil. Campinas: UNICAMP; 2005. p. I-5.

I I Santos E, Oliveira J. Utilização de óleo de jojoba como coletor alternativo na flotação seletiva entre apatita e calcita. In: Painel PEMM, 2012; Rio de Janeiro. Rio de Janeiro: UFRJ; 20I2. p. I-2.

I2 REMAPE: Rede Macaúba de Pesquisa. Viçosa: Universidade Federal de Viçosa; 2016 [acesso em 04 Jul 20 I6]. Disponível em: http://www.macauba.ufv.br/.

I 3 Silva AC, Silva EMS, Rocha TWP. Microflotação de apatita utilizando óleo da castanha de macaúba (Acrocomia Aculeata) como coletor. Tecnologica em Metalurgia, Materiais e Mineração. 20 I5; I2(2): I46-I52.

14 Silva AC, Silva EMS, Silva TC, Alves BE. Apatite microflotation using pequi oil. Mineral Processing and Extractive Metallurgy: Transactions of the Institutions of Mining and Metallurgy: Section C. 20I5; I24(4):233-239.

Recebido em: II Jun. 2016

Aceito em: 4 Ago. 2016 